Check for updates

Cite this: RSC Adv., 2020, 10, 29745

\title{
Detection of tyrosine and monitoring tyrosinase activity using an enzyme cascade-triggered colorimetric reaction $\uparrow$
}

\begin{abstract}
Huei-Yu Chen and Yi-Chun Yeh (DD*
The aromatic amino acid tyrosine is an essential precursor for the synthesis of catecholamines, including LDOPA, tyramine, and dopamine. A number of metabolic disorders have been linked to abnormal tyrosine levels in biological fluids. In this study, we developed an enzyme cascade-triggered colorimetric reaction for the detection of tyrosine, based on the formation of yellow pigment (betalamic acid) and red fluorometric betaxanthin. Tyrosinase converts tyrosine to L-DOPA, and DOPA-dioxygenase catalyzes oxidative cleavage of L-DOPA into betalamic acid. Response is linear for tyrosine from 5 to $100 \mu \mathrm{M}$, and the detection limit (LOD) is $2.74 \mu \mathrm{M}$. The enzyme cascade reaction was applied to monitor tyrosinase activity and tyrosinase inhibition assays. Lastly, the performance of the proposed biosensor proved successful in the analysis of urine samples without the need for pre-treatment.
\end{abstract}

Received 26th June 2020

Accepted 30th July 2020

DOI: 10.1039/d0ra05581f

rsc.li/rsc-advances

Tyrosinase converts Tyr to L-DOPA; i.e., the substrate of

\section{Introduction}

Tyrosine (Tyr) is an aromatic amino acid critical to the synthesis of compounds such as neurotransmitters and melanin.1,2 Abnormal Tyr concentrations in plasma/urine can also be used as a biomarker in the detection of various diseases, such as alkaptonuria, tyrosinemia, and liver disease..$^{3-5}$ Therefore, an easy quantification assay of Tyr is important and necessary.

Researchers have developed numerous methods for the quantification of Tyr, including fluorometric methods, ${ }^{6-10}$ highperformance liquid chromatography, ${ }^{11}$ electrochemical detection, ${ }^{12-14}$ colorimetric assays, ${ }^{15}$ and chemiluminescence; ${ }^{16}$ however, implementing such methods is expensive and complex. In this study, we sought to facilitate the quantification of Tyr for diagnostic purposes by developing an enzyme-based colorimetric biosensor to reduce the possible interferences from catecholamine metabolites. The approach has proven highly effective, particularly in real-time detection.

Tyrosinase is a copper-containing oxidase, capable of catalyzing the hydroxylation of monophenol to catechol, and the subsequent oxidation to $o$-quinone, ${ }^{17}$ which carries a chromophoric group suitable as a biosensor readout. ${ }^{18}$ However, tyrosine and tyrosine analogs (e.g. tyramine) are also substrates of tyrosinase, and $o$-quinone is susceptible to non-enzymatic reactions resulting in the spontaneous formation of melanin..$^{19}$ We therefore developed a sequential cascade reaction involving multiple enzymes to serve as a signal filter and amplifier. ${ }^{20,21}$

Department of Chemistry, National Taiwan Normal University, Taiwan. E-mail: yichuny@ntnu.edu.tw

$\dagger$ Electronic supplementary information (ESI) available. See DOI: $10.1039 /$ d0ra05581f
DOPA-dioxygenase (DOD). Note that DOD is an oxidase that opens the cyclic ring between carbon 4 and 5 on L-DOPA, resulting in the synthesis of yellow betalamic acid, ${ }^{22-24}$ which is a precursor of betalains/betaxanthins used as readouts of optical biosensors (Scheme 1). ${ }^{25-27}$ Thus, we developed an enzyme cascade-triggered colorimetric biosensor for Tyr involving the synthesis of yellow betalamic acid and red betaxanthins products. We then evaluated the proposed biosensor in terms of Tyr detection.

\section{Experimental methods}

\subsection{Chemicals}

2-Aminophthalic acid (2-AIPA), epinephrine, glucose oxidase, lysozyme, norepinephrine, para-aminobenzoic acid (PABA), tyrosinase, and tryptophan were purchased from Sigma-Aldrich. L-Ascorbic acid (AA), carbenicillin, iron(II) sulfate, isopropyl $\beta$-D1-thiogalactopyranoside (IPTG), and protease inhibitor cocktail were purchased from Amresco. Glutamic acid (Glu), L-DOPA, and phenylalanine (Phe) were purchased from ACROS. paraPhenylenediamine and tyramine were purchased Alfa Aesar. Calcium chloride, magnesium chloride hexahydrate, potassium chloride, and tyrosine were purchased from Merck. Sodium chloride and trypsin were purchased from VWR Life Science and Promega, respectively. Artificial urine (AU) was purchased from GE Healthcare Life Sciences.

\subsection{Expression and purification of DOPA-dioxygenase}

E. coli BL21 (DE3) (Invitrogen) carrying pET21a-DOD-histag plasmid was inoculated in LB medium containing $1 \mathrm{mM}$ of 


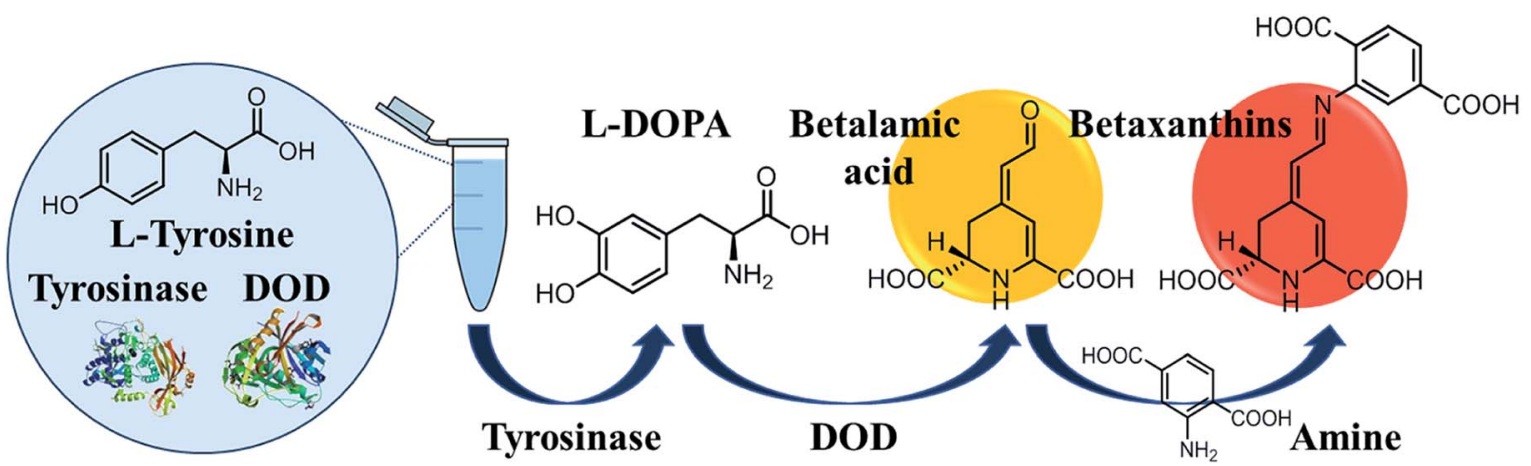

Scheme 1 Enzyme cascade-triggered colorimetric reaction for Tyr analysis based on the synthesis of betalamic acid and betaxanthins.

carbenicillin at $37^{\circ} \mathrm{C}$ for $16-18 \mathrm{~h}$. Then, the overnight culture was diluted in fresh LB $(1: 100 ; \mathrm{v} \%)$ with $1 \mathrm{mM}$ of carbenicillin and incubated at $37^{\circ} \mathrm{C}$ until O.D. 600 reached 0.4-0.6, and $1 \mathrm{mM}$ of IPTG was used to induce protein expression at $18^{\circ} \mathrm{C}$ for $22 \mathrm{~h}$. Next, the pellets were lysed in lysis buffer containing protease inhibitor by sonication after the harvest of the cells by centrifugation at $9000 \mathrm{rpm}$ and $4{ }^{\circ} \mathrm{C}$ (lysis buffer containing $25 \mathrm{mM}$ of Tris- $\mathrm{HCl}$ with $200 \mathrm{mM}$ of $\mathrm{NaCl}$ ). Then, the lysate was centrifuged at $9000 \mathrm{rpm}$ and $4{ }^{\circ} \mathrm{C}$ for $1 \mathrm{~h}$ to remove cell debris. After that, HisPur ${ }^{\mathrm{TM}}$ Ni-NTA Resin Spin Columns (Thermo Scientific) were used to purify the proteins in the supernatant, which was filtered through a $0.22 \mu \mathrm{m}$ syringe filter, and the purified proteins were dialyzed against $25 \mathrm{mM}$ of phosphate buffer with $100 \mathrm{mM}$ of $\mathrm{NaCl}$ (pH 6.8) for $24 \mathrm{~h}$.

\subsection{Detection of tyrosine by the enzyme cascade-triggered colorimetric reaction and interference study}

This colorimetric biosensor for Tyr analysis was incubated with various concentrations of Tyr in the reaction medium containing sodium phosphate buffer $(25 \mathrm{mM})$ with $\mathrm{NaCl}(100 \mathrm{mM})(\mathrm{pH}$ 6.8), iron(II) sulfate $(0.5 \mathrm{mM})$, ascorbic acid $(10 \mathrm{mM}),{ }^{27}$ tyrosinase $(224 \mu \mathrm{M})$, and DOD $(12.5 \mu \mathrm{M})$. The optical signal was detected by measuring the absorption spectroscopy in 96-well plates in a Synergy HT microplate reader (BioTek, Winooski, VT) after the enzyme cascade-triggered reaction at room temperature for $0.5 \mathrm{~h}$. The reaction medium without Tyr was mixed with tyramine, norepinephrine, epinephrine, phenethylamine, phenylalanine, tryptophan and ascorbic acid at $100 \mu \mathrm{M}$ for interference tests respectively.

\subsection{Characterization and quantification of betaxanthins}

The reaction solutions containing Tyr at various concentrations were mixed with $10 \mathrm{mM}$ primary amines. The fluorescence intensity of betaxanthins was detected on F-7000 fluorescence spectrophotometer (HITACHI) after the enzymatic conversion of Tyr to betalamic acid and further the non-enzymatic condensation of betalamic acid with amines to synthesis betaxanthins at room temperature for $1 \mathrm{~h}$.

\subsection{Preparation of artificial urine samples}

Artificial urine was diluted 10-fold with sodium phosphate buffer before all measurements.

\section{Results and discussion}

\subsection{Characterization and optimization of betalamic acid production}

Using absorption spectroscopy, we began by determining the optimal conditions for the enzyme cascade system based on the detection of the yellow betalamic acid. Absorption intensity at $430 \mathrm{~nm}$ was shown to increase with the concentration of Tyr. The colorimetric sensor system was also assessed under various
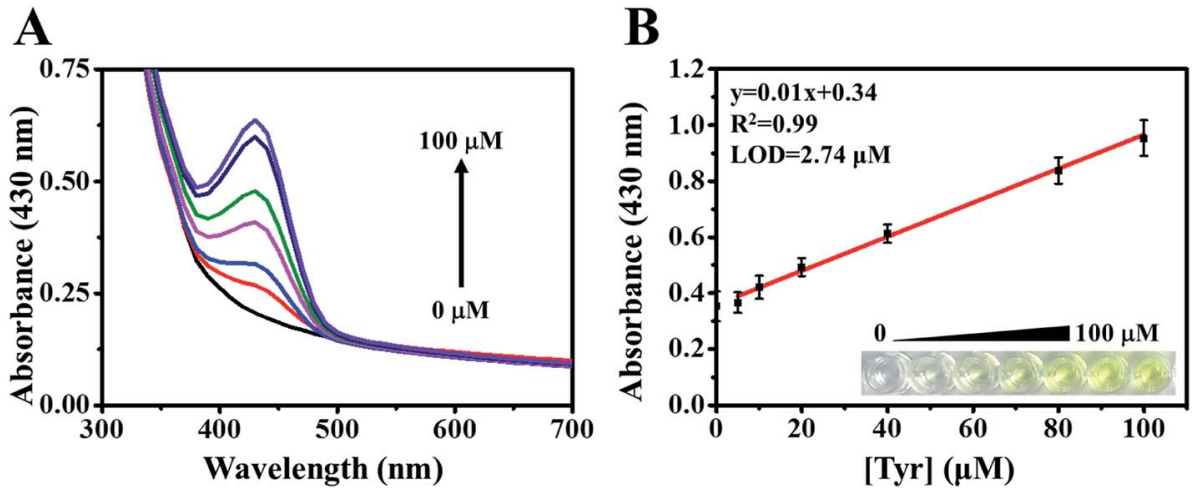

Fig. 1 (A) Absorption spectra of reaction mixtures with various concentrations of Tyr $(0,5,10,20,40,80$, and $100 \mu M)$. (B) The linear range for Tyr was between 5 and $100 \mu \mathrm{M}$, and LOD was $2.74 \mu \mathrm{M}$. The corresponding photograph under the natural light in the presence of Tyr. 
A

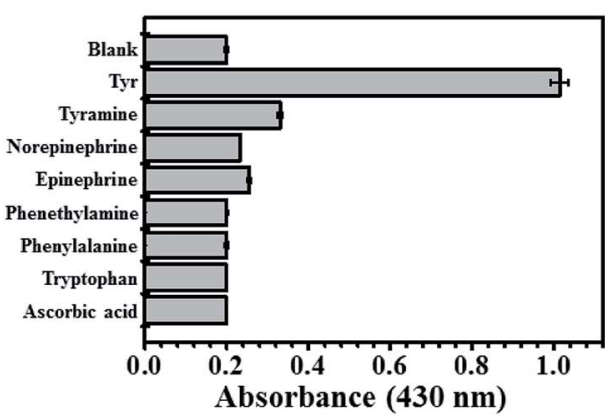

B

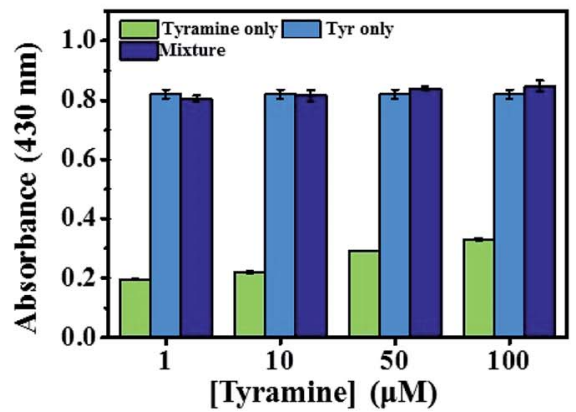

Fig. 2 (A) The selectivity of the enzyme cascade-based system in the presence of other substrates at $100 \mu \mathrm{M}$ was investigated. The intensity of absorbance at $430 \mathrm{~nm}$ after $0.5 \mathrm{~h}$ reaction was recorded. (B) The intensities of $\lambda_{430}$ in the co-presence of Tyr at $100 \mu \mathrm{M}$ and $1 / 10 / 50 / 100 \mu \mathrm{M}$ of tyramine.

$\mathrm{pH}$ values in order to determine the optimal buffer conditions (Fig. S1A $\dagger$ ). Our results indicate that $\mathrm{pH} 6.8$ is ideal for this system.

We then sought to optimize the concentrations of tyrosinase and DOD in the proposed system. We began by varying the concentration of tyrosinase between 0 to $224 \mu \mathrm{M}$ in the presence of DOD $(8 \mu \mathrm{M})$ and Tyr $(50 \mu \mathrm{M})$ following incubation for a period of $0.5 \mathrm{~h}$. We observed a notable increase in absorption intensities at $430 \mathrm{~nm}$ at the highest tyrosinase concentration of 224 $\mu \mathrm{M}$ (Fig. S1B $\dagger$ ). We then increased the concentrations of DOD from 8 to $20 \mu \mathrm{M}$ in the presence of tyrosinase $(224 \mu \mathrm{M})$ and $\mathrm{Tyr}$ $(50 \mu \mathrm{M})$ (Fig. S1C $\dagger$ ). We did not observe a notable increase in $\lambda_{430}$ signal intensity when the DOD concentration was increased beyond $12.5 \mu \mathrm{M}$. Thus, throughout the remainder of the study, the enzyme cascade-based biosensor was implemented using $224 \mu \mathrm{M}$ of tyrosinase and $12.5 \mu \mathrm{M}$ of DOD.

We closely monitored the $\lambda_{430}$ signal intensity throughout the time-course of the reaction (Fig. S1D $\dagger$ ). Note that the initial increase in intensity plateaued within $1 \mathrm{~h}$. Thus, in all subsequent experiments, we recorded the absorption intensity at $430 \mathrm{~nm}$ after incubation for $0.5 \mathrm{~h}$ in order to enable rapid detection with reasonable incubation. The results suggest the completion time of proposed sensor is comparable to methods which are commonly used.

\subsection{Determining substrate specificity of the tyrosine sensing system}

Under optimal experimental conditions, a dramatic increase in the absorption intensity of betalamic acid was observed upon the concentration of Tyr increased (Fig. 1A). The absorption intensity of betalamic acid was shown to increase linearly with the addition of Tyr at concentrations of 5 to $100 \mu \mathrm{M}\left(R^{2}=0.99\right)$ (Fig. 1B). According to the $3 \mathrm{sb} /$ slope, the detection limit (LOD) was $2.74 \mu \mathrm{M}$ (sb: standard deviation of background and the slope in the calibration plot).

The selectivity of the biosensor was evaluated by mixing the reaction medium with interfering substrates, including structural analogs (tyramine, norepinephrine, epinephrine, and phenethylamine) and metabolic intermediates (phenylalanine, tryptophan, and ascorbic acid) at concentrations of $100 \mu \mathrm{M}$, respectively. It was found that tyramine (structurally similar to Tyr) subtlety increased the intensity of the $\lambda_{430}$ signal (Fig. 2A). Therefore, to test the response to interference in the co-
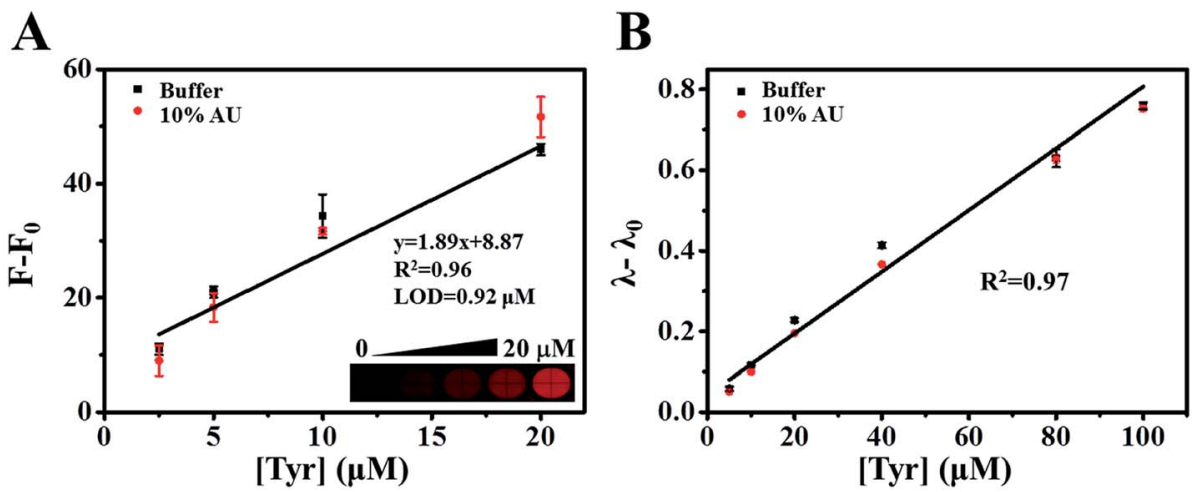

Fig. 3 The intensity of (A) the red fluorescence intensities of 2-AIPA-betaxanthin $\left(F-F_{0}\right)$ and (B) $\lambda-\lambda_{0}$ for betalamic acid was observed as a linear function with the concentrations of $\operatorname{Tyr}\left(F_{0}\right.$ and $\lambda_{0}$ represent the background absorbance and fluorescence of blank matrices). (A) The linear ranges for Tyr was from 2.5 to $20 \mu \mathrm{M}$, and LOD was $0.92 \mu \mathrm{M}$. The effectiveness of enzyme cascade-based biosensor in buffer and $10 \%$ artificial urine matrix was compared (red dots). Inset: the corresponding fluorescence photograph in the presence of Tyr. 
presence of tyramine and Tyr, we compared the enhanced intensities of $\lambda_{430}$ with that of a single substrate and a mixture of the two. In addition, we studied the responses using various tyramine to Tyr ratios $(1,10,50,100 \mu \mathrm{M})$ and Tyr $(100 \mu \mathrm{M})$. As shown in Fig. 2B, the absorbance intensity at $430 \mathrm{~nm}$ has not interfered. Thus, the effect of tyramine can be considered negligible.

\subsection{Stability of the enzyme cascade-based sensor}

Stability is a crucial concern in assessing any biosensor. In this study, we compared the stability of the proposed enzyme cascade-triggered biosensor in the detection of Tyr (based on the formation of betalamic acid) and the tyrosinase system (based on the formation of dopachrome). Tyrosinase could lead to the hydroxylation of Tyr to L-DOPA and subsequently catalyze the oxidation of L-DOPA to dopachrome leading to an increase in absorption at $475 \mathrm{~nm}$ (Fig. S2A $\dagger$ ). ${ }^{17,18}$ We therefore added Tyr (at various concentrations) to a reaction medium containing phosphate buffered saline ( $\mathrm{pH} 7.4$ ) and $224 \mu \mathrm{M}$ tyrosinase. We then observed the stability of the two systems at regular intervals by measuring $\lambda_{430}$ of betalamic acid and $\lambda_{475}$ of dopachrome in the presence of Tyr at a concentration of $100 \mu \mathrm{M}$ (Fig. $\mathrm{S} 2 \mathrm{~B} \dagger$ ). The intensity of $\lambda_{475}$ (tyrosinase system biosensor) presented a steady decrease after $7 \mathrm{~h}$ incubation. By contrast, the intensity of $\lambda_{430}$ (enzyme cascade-triggered biosensor) remained constant for more than $24 \mathrm{~h}$.

Again, the co-presence of tyramine and Tyr was examined by monitoring intensities of $\lambda_{475}$ with that of a single substrate and a mixture of the two using conventional tyrosinase assays. The intensity of $\lambda_{475}$ was shown enhancement than normal in the copresence of tyramine at $10 \mu \mathrm{M}$ (Fig. S2C $\dagger$ ), indicating interference can occur for the samples containing tyramine greater than $10 \mu \mathrm{M}$.

\subsection{Condensation of betalamic acid with amines}

It has been shown that the color of betanin depends on the structural motifs in the cyclo-DOPA moiety of the pigments. ${ }^{28,29}$ We therefore mixed betalamic acid with $10 \mathrm{mM}$ of primary amines phenylalanine (Phe), glutamic acid (Glu), para-aminobenzoic acid (PABA), and 2-aminoterephthalic acid (2-AIPA), which respectively present green (Phe-, Glu-) and red (PABA-, 2AIPA-) fluorescence. ${ }^{27}$ We then monitored the green and red fluorescence intensity of betaxanthins at various time points (Fig. S3A and S3B $\dagger$ ). The green fluorescence intensity plateaued after $21 \mathrm{~h}$; however, the red fluorescence intensity peaked after $4 \mathrm{~h}$. To increase detection efficiency, we selected 2-AIPA-betaxanthin for the quantification of Tyr. The optimal concentration for 2-AIPA was examined in Fig. S3C. $\dagger$ The red fluorescence intensities of 2-AIPA-betaxanthin was shown to increase linearly with the concentration of Tyr (from 2.5 to $20 \mu \mathrm{M}$ ) with $R^{2}=$ 0.96 (Fig. 3A).

\subsection{The colorimetric biosensor for tyrosine detection using artificial urine matrix}

Normally, the concentration of Tyr in healthy individuals ranges from 30 to $120 \mu \mathrm{M} ;^{30}$ however, it can exceed $200 \mu \mathrm{M}$ in the
A
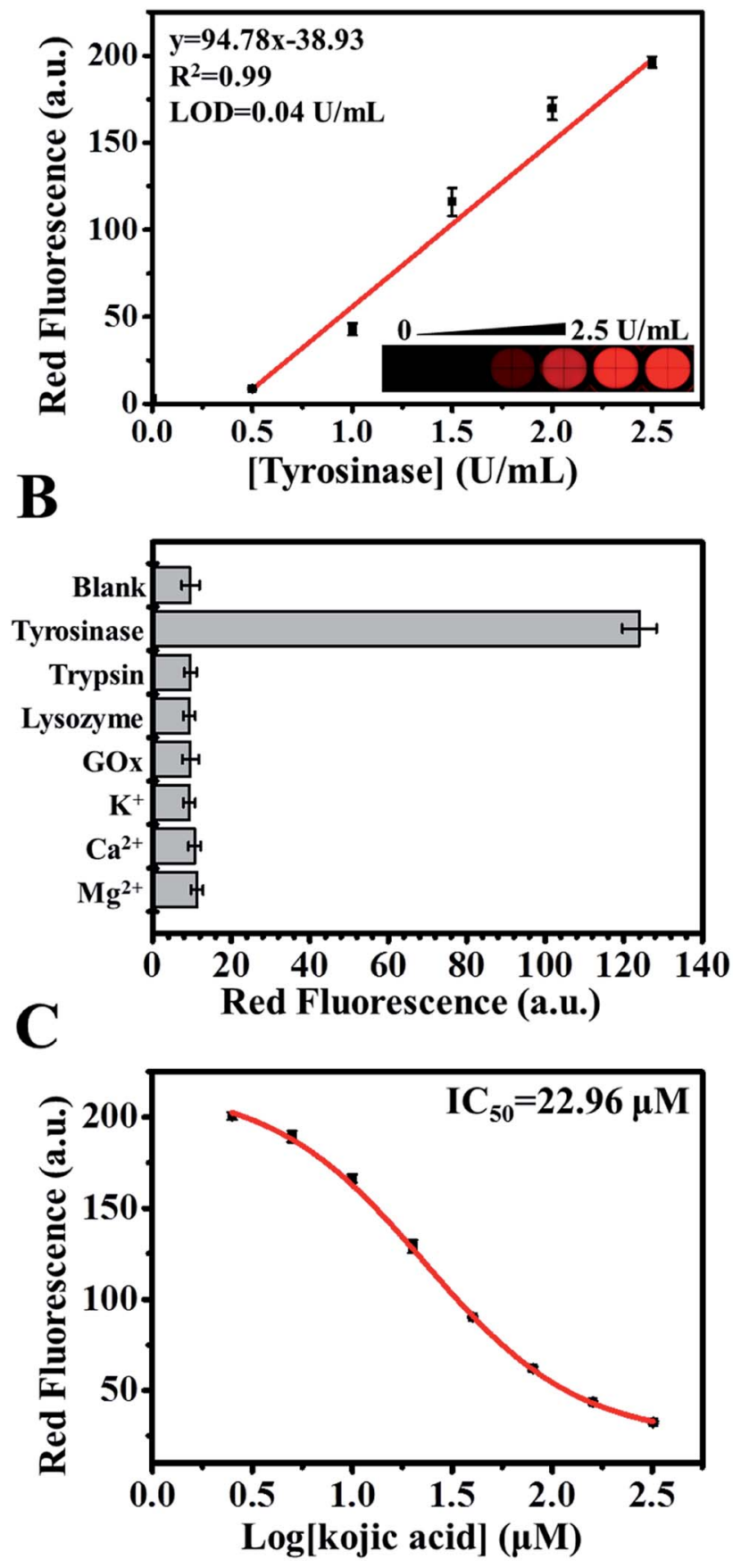

Fig. 4 (A) The linear relationships between the red fluorescence intensities of 2-AIPA-betaxanthin and tyrosinase at various concentrations. Inset: the corresponding fluorescence photograph in the presence of tyrosinase. (B) The selectivity of the proposed enzyme cascade-based system in the presence of various substrates (tyrosinase, trypsin, lysozyme, GOx: $\left.6 \mathrm{U} \mathrm{mL}^{-1} ; \mathrm{K}^{+}, \mathrm{Ca}^{2+}, \mathrm{Mg}^{2+}: 100 \mu \mathrm{M}\right)$ was investigated. (C) The red fluorescence intensities of 2-AIPA-betaxanthin in the presence of Tyr $(1000 \mu \mathrm{M})$, tyrosinase $\left(9 \mathrm{U} \mathrm{mL}^{-1}\right)$, and kojic acid at various concentrations.

biological fluids of patients with tyrosine metabolism disorders. We therefore tested the effectiveness of the proposed enzyme cascade-based biosensor using 10\% diluted artificial urine 
spiked with various concentrations of Tyr. Our results revealed that the linearity, dynamic range, and sensitivity of fluorescence and absorbance intensity in complex matrices were similar to those in the buffer system (Fig. 3A and B). The calculated relative standard deviation (RSD) and recoveries using standard curves were obtained (Tables S1 and S2 $\dagger$ ). The accuracy ranges from $88-101 \%$ and $100-120 \%$ based on absorbance and fluorescence assays, respectively. This is a clear demonstration that this proposed method could be applied to the detection of Tyr in biological specimens.

\subsection{Monitoring tyrosinase activity using the enzyme cascade reaction}

Next, we employed the enzyme cascade reaction for the visual observation of tyrosinase activity. We first determined the optimal concentration of Tyr for the enzyme cascade reaction. We began by varying the concentration of Tyr between 125 to $1000 \mu \mathrm{M}$ in the presence of DOD $(12.5 \mu \mathrm{M})$ and tyrosinase $(1.5 \mathrm{U}$ $\mathrm{mL}^{-1}$ ) following incubation for a period of $1 \mathrm{~h}$. As shown in Fig. S4A, $\uparrow$ the intensities of $\lambda_{430}$ were significantly increased when $1000 \mu \mathrm{M}$ Tyr was introduced. Under optimal experimental conditions, a good linear relationship was observed between the intensities of $\lambda_{430}$ and tyrosinase concentrations (Fig. S4B $\dagger$ ). The red fluorescence intensities of 2-AIPA-betaxanthin was recorded as well to determine the tyrosinase activity (Fig. 4A). The corresponding regression coefficient is 0.99 , and the LOD was $0.04 \mathrm{U} \mathrm{mL}^{-1}$. The selectivity and activity of the proposed sensor was investigated by using the interfering substances including trypsin, lysozyme, glucose oxidase (GOx), and metal ions (Fig. 4B). There is no noticeable change of red fluorescence intensity in the presence of interfering substances.

The development of synthetic and naturally occurring tyrosinase inhibitors has attracted an increased research in the medical, agricultural, and cosmetic industries. Among many naturally occurring tyrosinase inhibitors, kojic acid has been intensively studied. We sought to examine the effectiveness of the proposed sensors in screening tyrosinase inhibitors by using kojic acid as a model compound. The red fluorescence of 2-AIPA-betaxanthin was recorded in the presence of Tyr (1000 $\mu \mathrm{M})$, tyrosinase (9 $\mathrm{U} \mathrm{mL}^{-1}$ ), and kojic acid at various concentrations (Fig. 4C). The calculated $\mathrm{IC}_{50}$ value was $22.96 \mu \mathrm{M}$, which is consistent with the previous reports. ${ }^{31,32}$

\section{Conclusions}

In this study, we have developed an enzyme cascade-triggered colorimetric biosensor for the quantification of Tyr. Tables S3 and $\mathrm{S} 4 \uparrow$ present a comparison with several sensors in terms of the linear ranges, LOD, and analysis duration. The formation of dopachrome using tyrosinase is commonly used for the detection of Tyr. However, tyrosinase shows both the hydroxylase and the oxidase activities. To overcome the specificity issues, we developed cascade-triggered sensor, which has satisfactory sensitivity and selectivity to Tyr. The proposed biosensor also proved effective in the detection of Tyr in realistic samples, and makes it possible to detect Tyr using the naked eye. Two types of signals including colorimetric and fluorescent method were examined. Although fluorescent method of 2-AIPA-betaxanthin showed a better sensitivity than colorimetric method, the analysis duration is relative longer than the colorimetric method. These findings suggest that the effectiveness and simplicity of the proposed scheme make it an attractive strategy for the development of biosensors for Tyr and tyrosinase analysis.

\section{Conflicts of interest}

There are no conflicts to declare.

\section{Acknowledgements}

This work was funded by the Ministry of Science and Technology of Taiwan under the project number 107-2113-M-003013-MY3.

\section{References}

1 J. P. Greenstein, Chemistry of the amino acids, 1961.

2 C. R. Scriver, The metabolic \& molecular bases of inherited disease, McGraw-Hill, New York; Montreal, 2001.

3 A. Chakrapani and E. Holme, Inborn metabolic diseases, 2006, pp. 233-243.

4 K. Michitaka, A. Hiraoka, M. Kume, T. Uehara, S. Hidaka, T. Ninomiya, A. Hasebe, Y. Miyamoto, M. Ichiryu and T. Tanihira, Hepatol. Res., 2010, 40, 393-398.

5 J. B. Mistry, M. Bukhari and A. M. Taylor, Rare Dis., 2013, 1, e27475.

6 J. A. Ambrose, Clin. Chem., 1974, 20, 505-510.

7 F. Wang, Y. Qing and Y.-X. Ci, Anal. Lett., 1992, 25, 14691478.

8 Y. Li, N. Cai, M. Wang, W. Na, F. Shi and X. Su, RSC Adv., 2016, 6, 33197-33204.

9 X. Qin, Y. Lu, M. Bian, Z. Xiao, Y. Zhang and Y. Yuan, Anal. Chim. Acta, 2019, 1091, 119-126.

10 M. Halawa, J. Lai and G. Xu, Mater. Today Nano, 2018, 3, 927.

11 Y. Ishii, M. Iijima, T. Umemura, A. Nishikawa, Y. Iwasaki, R. Ito, K. Saito, M. Hirose and H. Nakazawa, J. Pharm. Biomed. Anal., 2006, 41, 1325-1331.

12 L. Jiang, S. Gu, Y. Ding, D. Ye, Z. Zhang and F. Zhang, Colloids Surf., B, 2013, 107, 146-151.

13 P. Kanchana, N. Lavanya and C. Sekar, Mater. Sci. Eng., C, 2014, 35, 85-91.

14 Y. Wang, C. Xiong, H. Qu, W. Chen, A. Ma and L. Zheng, J. Electroanal. Chem., 2017, 799, 321-326.

15 G. Vyas, S. Bhatt, M. K. Si, S. Jindani, E. Suresh, B. Ganguly and P. Paul, Spectrochim. Acta, Part A, 2020, 230, 118052.

16 S. Li, M. Xing, H. Wang, L. Zhang, Y. Zhong and L. Chen, RSC Adv., 2015, 5, 59286-59291.

17 T. Hasegawa, Int. J. Mol. Sci., 2010, 11, 1082-1089.

18 A. S. Saini, J. Kumar and J. S. Melo, Anal. Chim. Acta, 2014, 849, 50-56. 
19 P. Agarwal, M. Singh, J. Singh and R. Singh, Applied Microbiology and Bioengineering, 2019, pp. 3-19.

20 Y. Zhang, S. Tsitkov and H. Hess, Nat. Commun., 2016, 7, 13982.

21 J. Zhao, S. Wang, S. Lu, X. Bao, J. Sun and X. Yang, Anal. Chem., 2018, 90, 7754-7760.

22 N. Sasaki, Y. Abe, Y. Goda, T. Adachi, K. Kasahara and Y. Ozeki, Plant Cell Physiol., 2009, 50, 1012-1016.

23 F. Gandía-Herrero and F. García-Carmona, Trends Plant Sci., 2013, 18, 334-343.

24 T. Nakatsuka, E. Yamada, H. Takahashi, T. Imamura, M. Suzuki, Y. Ozeki, I. Tsujimura, M. Saito, Y. Sakamoto and N. Sasaki, Sci. Rep., 2013, 3, 1970.

25 P.-H. Chen, C. Lin, K.-H. Guo and Y.-C. Yeh, RSC Adv., 2017, 7, 29302-29305.
26 Y.-K. Lin and Y.-C. Yeh, Anal. Chem., 2017, 89, 11178-11182. 27 Y.-C. Chou, C.-I. Shih, C.-C. Chiang, C.-H. Hsu and Y.-C. Yeh, Sens. Actuators, B, 2019, 126717.

28 F. Gandía-Herrero, J. Escribano and F. García-Carmona, Planta, 2010, 232, 449-460.

29 P. S. Grewal, C. Modavi, Z. N. Russ, N. C. Harris and J. E. Dueber, Metab. Eng., 2018, 45, 180-188.

30 K. Nakamura, S. Matsumoto, H. Mitsubuchi and F. Endo, Pediatr. Int., 2015, 57, 37-40.

31 G. Liu, J. Zhao, S. Lu, S. Wang, J. Sun and X. Yang, ACS Sens., 2018, 3, 1855-1862.

32 T.-I. Kim, J. Park, S. Park, Y. Choi and Y. Kim, Chem. Commun., 2011, 47, 12640-12642. 